\title{
Adaptive Locks: Combining Transactions and Locks for Efficient Concurrency
}

\author{
Takayuki Usui ${ }^{\mathrm{a}, 1}$, Reimer Behrends ${ }^{\mathrm{a}, 2}$, Jacob Evans $^{\mathrm{b}}$, Yannis Smaragdakis ${ }^{\mathrm{b}, *}$ \\ ${ }^{a}$ Department of Computer and Information Science, University of Oregon, Eugene, OR 97403, USA \\ ${ }^{b}$ Department of Computer Science, University of Massachusetts, Amherst, MA 01003, USA
}

\begin{abstract}
Transactional memory is being advanced as an alternative to traditional lock-based synchronization for concurrent programming. Transactional memory simplifies the programming model and maximizes concurrency. At the same time, transactions can suffer from interference that causes them to often abort, from heavy overheads for memory accesses, and from expressiveness limitations (e.g., for I/O operations). In this paper we propose an adaptive locking technique that dynamically observes whether a critical section would be best executed transactionally or while holding a mutex lock. The critical new elements of our approach include the adaptivity logic and cost-benefit analysis, a low-overhead implementation of statistics collection and adaptive locking in a full $\mathrm{C}$ compiler, and an exposition of the effects on the programming model. In experiments with both micro- and macro-benchmarks we found adaptive locks to consistently match or outperform the better of the two component mechanisms (mutexes or transactions). Compared to either mechanism alone, adaptive locks often provide 3-to-10x speedups. Additionally, adaptive locks simplify the programming model by reducing the need for fine-grained locking: with adaptive locks, the programmer can specify coarse-grained locking annotations and often achieve fine-grained locking performance due to the transactional memory mechanisms.
\end{abstract}

\footnotetext{
${ }^{*}$ Corresponding author

Email addresses: tusui@uoregon.edu (Takayuki Usui), behrends@cs . uoregon. edu (Reimer Behrends), jmevans@cs.umass.edu (Jacob Evans), yannis@cs. umass .edu (Yannis Smaragdakis)

${ }^{1}$ Currently at 3Di Inc.

${ }^{2}$ Currently at University of St. Andrews, Scotland
} 


\section{Introduction}

Multi-core processors are turning shared-memory parallelism into the default model of computation for mainstream software development. Although there are ways to take advantage of such parallelism through different high-level paradigms (e.g., stream processing [1] or message passing [2]) Explicit multi-threading remains the most direct way to program parallel systems. In the multi-threaded programming world, interference between threads is a major issue and results in hard-to-trace defects such as race conditions or deadlocks. Traditionally, programmers have coordinated threads using programming patterns based on mutual-exclusion (mutex) locks.

In recent years, an alternative model has been proposed for thread coordination. Transactional memory (TM) replaces mutexes and condition variables with "atomic" blocks of code, that are meant to execute as if all other threads had stopped running during the execution of the atomic block. TM has intrigued both software and hardware designers, and many major processor manufacturers have already announced support for TM in upcoming architectures. The advantage of TM is twofold: First, it offers a higher-level programming model by obviating the need for stating which locks to acquire. This means that code is more composable: Callers do not need to know which locks their callees hold, and writing code does not require global knowledge of which locks are used by possibly interfering threads. The possibility of low-level deadlock is also avoided, as there is no potential for the programmer to erroneously specify circular lock dependencies. Furthermore, TM does not require fine-grained delineation of critical sections in order to achieve high concurrency. Most TM implementations allow threads to proceed unless they interfere on the same shared memory data. In contrast, mutex locks conservatively prevent threads from proceeding if they need to acquire the same lock, even if they never access the same data.

The TM approach is not free of disadvantages, however. Transactions eliminate deadlock, but replace it with possible livelock or slower progress: Interfering threads can cause each other's transactions to abort and retry. Furthermore, transactions cannot easily support irreversible operations, such as $\mathrm{I} / \mathrm{O}$, despite several proposals in this direction [3-6]. Finally, when transactions are implemented in software they can suffer 
from high overheads during the execution of atomic blocks: Every shared memory read and write operation needs to be trapped and treated specially. The overheads have led some authors to even claim that software TM is "only a research toy" [7].

In this paper, we present adaptive locks: a synchronization mechanism combining locks and transactions for best performance. In our approach, the programmer specifies critical sections, which can be executed either with mutual exclusion or atomically as transactions. For instance a critical section

atomic (11) $\{\ldots\}$

is equivalent to either

atomic $\{\ldots\}$

(when the system executes in transaction mode) or

lock(11); ... unlock(11);

(when the system executes in mutex mode). At any point in time, all critical sections that use the same lock, 11, have to execute in the same mode.

The decision to execute in mutex mode or in transaction mode depends on the observed behavior of the critical section, namely on the nominal contention (how many threads are blocked on the lock when in mutex mode), the actual contention (how many times each transaction retries when in transaction mode), and the transactional overhead (how much slower is the critical section when in transaction mode compared to mutex mode). Our adaptive locks compute these three factors dynamically during the program's execution and combine them for an accurate cost-benefit analysis, as described in Section 2. We present techniques for performing this computation highly efficiently. The overall adaptive lock implementation imposes very low overhead compared to either a regular mutex lock or a transaction.

What adaptive locks achieve is the ability to dynamically switch concurrency mechanisms depending on execution conditions. A single code base (e.g., a library implementation of a general data structure, such as a hash table or tree) can be used in environments with high or low contention and always achieve optimal performance. For example, a program could contain two tree structures, both implemented by the same 
code, but one of them being large and accessed by many threads, while the other being small or only infrequently accessed concurrently. With adaptive locks, unnecessary overheads due to concurrency mismatch will be avoided for both data structures.

The adaptive locks programming model resembles mutex locks more than it does transactions. For instance, the deadlock-freedom and composability guarantees of transactions are not preserved, since our critical sections may execute in mutex lock mode. It is, therefore, important to ask, "are adaptive locks just an optimized implementation of locks?" Based on the benefits observed in our evaluation, we argue that the practical impact of adaptive locks is much more than that. We believe that adaptive locks significantly change the programming model for concurrency. Adaptive locks allow the programmer to concentrate only on coarse-grained locking approaches, instead of trying to achieve more performance by introducing error-prone fine-grained locks. The performance of fine-grained locks is then often fully recovered automatically by employing the TM mechanism when appropriate. All our benchmark measurements are implemented with very coarse-grained lock annotations (often a single global lock, which trivially has good composability and deadlock-freedom properties), yet still achieve significant performance improvements. (Indeed, such coarse-grained locks can also be automatically inferred for correctness-e.g., [8].) Thus, adaptive locks encourage programmers to use locks at whichever level of abstraction correctness is easy to establish, and not at the granularity needed for performance.

Some of our work's closest relatives in the research literature are Rajwar and Goodman's lock elision [9] and Welc et al.'s transactional monitors [10]. (There is more related work and we discuss it in detail in Section 6.) Lock elision is a hardware technique for (effectively) implementing locks as low-level transactions, but with no clear adaptive cost-benefit model, as the one we introduce. Welc et al.'s transactional monitors implement locks optimistically as soon as the monitor encounters contention. Again, there is no dynamic cost-benefit model for the two modes of execution, or a possibility of reverting back to locks if the TM mechanism turns out to be inefficient. Welc et al. acknowledge the need for more adaptive solutions, which our work provides. Finally, the work in this paper is an evolution and realization of the non-blocking locks idea that we presented in an earlier position paper [11]. Overall, our concrete contributions are 
as follows:

- We present a highly efficient and effective implementation of the concept of adaptive locks. Our adaptive locks keep precise statistics on the behavior of the program, and dynamically adapt to it based on an online cost-benefit analysis, while introducing very low overhead: acquiring an adaptive lock is practically no more costly than acquiring a mutex lock. Importantly, this removes all performance arguments used in favor of locks and against Software Transactional Memory [7]: transactions are used only when they yield benefits, and incur no overhead otherwise. Thus, contrary to the assertion of Cascaval et al., Software Transactional Memory is much more than a "research toy": at the very least it is an excellent way to implement locks. We describe the optimizations responsible for our mechanism's efficiency-e.g., trading some inaccuracy in our statistics in exchange for shortening the critical path of lock acquisition and avoiding bottlenecks. Our implementation is in the form of a full C compiler, based on the CIL framework [12], and is freely available for download.

- We evaluate adaptive locks with several micro- and macro-benchmarks. Our evaluation shows that adaptive locks combine the performance benefits of mutex locks and transactions. In every case, the performance of adaptive locks closely matches the performance of the better of the two component mechanisms. This allows adaptive locks to achieve the highest possible performance not just for different applications, but also for different configurations of the same application. For instance, we demonstrate performance improvements of several factors for different degrees of hardware concurrency (e.g., 3x faster than TM for 2 processors, $3 \mathrm{x}$ faster than mutex locks for 64 processors).

Compared to either mutex locks or transactions alone, adaptive locks routinely achieve order-of-magnitude performance improvements by emulating the performance of the complementary mechanism. Adaptive locks occasionally outperform both component mechanisms at the same time, by up to $50 \%$, due to the varied contention behavior of different application phases. 


\section{Design and Adaptivity Logic}

We next discuss the concept of adaptive locks, as well as the cost-benefit logic that the locks implement in order to choose their optimal execution mode.

\subsection{Programming with Adaptive Locks}

Adaptive locks introduce syntax for a labeled atomic section. This is a block structured construct, headed by the keyword atomic with a label indicating which adaptive lock protects the code statement (usually a block statement) that follows. By convention, in this paper (as well as in our implementation) adaptive locks are declared as instances of type al_t, e.g.:

al_t lock1; ...

atomic (lock1) $\{\ldots / *$ critical section $* /\}$

The programmer is responsible for ensuring that the lock labels are "correct"-i.e., that the program will work correctly if all instances of atomic $(<l c k L b l>)$ are replaced by a regular mutex, Lock $(<l c k L b l>$ ). (We assume a block-structured mutex lock, with an unlock performed at the end of the block.) The programmer also has the obligation to ensure that the program is equally correct if all lock labels are dropped and all critical sections atomic $(<l c k L b l>)<s t m t>$ execute as transactions, atomic $<$ stmt $>$, in a conventional TM system (e.g., [13-15]). The reason is that transactions can have subtly different behavior from mutex locks $[11,14,16-18]$. Nevertheless, the topic of adaptive locks is orthogonal to such differences. For instance, one can implement an adaptive scheme with a TM system supporting single-global-lock semantics [17]. For weaker back-end TM systems, the programmer can ensure correctness of adaptive locks code under either mode by employing static separation (i.e., ensuring that data that are ever shared are always accessed under a lock [11, 19]) or dynamic separation [20] techniques. We will discuss this topic in Section 5, where we also offer a general condition for the semantic equivalence of transactions and mutexes. Note also that adaptive locks do not support transactional constructs that rely on retrying (such as an explicit retry or abort statement). Condition synchronization is supported explicitly as in a regular lock-based programming model, and not simulated using retry. 
The adaptive lock implementation is, thus, free to execute the critical section it protects either as a transaction or as a critical section protected by a mutex lock. ${ }^{3}$ As mentioned in the Introduction, we say that the adaptive lock is in transaction mode or in mutex mode, respectively. All critical sections associated with the same adaptive lock have to execute in the same mode at a given time. If a thread tries to acquire an adaptive lock and decides it wants to execute in a different mode than the current one, it marks the adaptive lock "in-transition" and waits until all current critical sections executing with this lock finish. (Clearly, there is more than one critical section executing only if the adaptive lock is in transaction mode.) While the lock is in-transition, no further mode switching decisions can be made. Furthermore, in the case of lock nesting, the mode of a nested adaptive lock cannot differ from the mode of a surrounding lock.

The reasons for switching the mode of an adaptive lock are either correctness- or performance-related. In the former case, if the lock is executing in transaction mode and an irreversible $\mathrm{I} / \mathrm{O}$ operation is called, the (outermost) critical section restarts in mutex mode. (Waiting on condition variables is also an irreversible action, so our adaptive locks revert to mutex mode execution when they encounter it inside a critical section.) The latter case captures the heuristic at the core of adaptive locks, for deciding when to switch modes in order to improve performance.

\subsection{Cost-Benefit Analysis}

The main reason for executing an adaptive lock in transaction mode is that mutex locks can exhibit false exclusion [21]. A single mutex lock is commonly used to protect a large amount of shared data—an approach known as coarse grained locking. In this way, multiple threads are blocked from accessing the data, even in cases when they would not really conflict. Programmers use coarse grained locking because it is often far easier than trying to correctly associate locks with smaller amounts of data. Several

\footnotetext{
${ }^{3}$ One can argue that the terms "transaction" and "mutex lock" refer to programming models, rather than implementation mechanisms. E.g., transactions can be implemented by a mechanism that guarantees exclusion, or mutex locks can be implemented speculatively. In this paper, we use the terms to refer to the implementation mechanisms overwhelmingly associated with them in common practice. We have found this to be best for communications purposes: when describing our work, listeners have been more likely to grasp it quickly if we explain it as a "mechanism adapting between mutex locks and transactions" rather than as a "mechanism adapting between speculative and non-speculative locks, where the speculation is implemented through TM techniques".
} 
domains and data structures (e.g., red-black trees) are notoriously difficult to code with a fine-grained locking discipline.

Therefore, the performance benefit of transactions is due to higher concurrency: More threads can execute the same critical section with transactions than with mutexes. Assuming that separate processors exist to run these threads, a net performance increase can result.

At the same time, executing an adaptive lock in transaction mode incurs high overheads when there is true contention on the data. In this case, different threads interfere with each other, preventing the successful commit of transactions. Therefore, transactions have to retry multiple times before they successfully commit, and the result is slower progress, or even livelock. The problem is solved when switching to mutex mode because the thread "reserves" the right to run up-front, thus making progress without interference.

A second factor hindering the performance of transaction mode is that, in puresoftware TM, there is typically a high overhead associated with executing a critical section transactionally. Software transactional memory (STM) systems [22] need to execute logging actions on each read or write operation of shared memory data. Depending on the design of the STM, the logged values are either used to update shared memory on transaction commit (redo-logging), or to revert shared memory to its previous state on transaction abort (undo-logging). ${ }^{4} \mathrm{~A}$ second overhead is due to the need to perform synchronization operations (e.g., acquiring locks associated with each written word) to ensure consistent memory writes. The need for logging actions and synchronization imposes a heavy overhead on shared memory operations and often slows down transaction mode execution of critical sections by a significant factor (e.g., 2-8x). Additionally, STM implementations often impose extra overheads for policy-specific reasons-e.g., re-validating the read set when a conflict is detected, incurring cost for aborting, etc.

\footnotetext{
${ }^{4}$ A few STM systems suffer no such overhead [23-25], by translating transactions into lock acquisitions and releases in a way that guarantees deadlock-freedom (and, thus, the transaction never needs to retry). The performance of such "auto-locking" systems depends crucially on (non-modular) compiler analysis or program annotation. No representative of this approach has yet achieved the same level of performance as standard STMs (pessimistic or optimistic) in a general-purpose, automatic setting.
} 
Therefore the adaptive lock analysis of whether to execute in transaction mode or mutex mode has to take into account three factors:

- Nominal contention $(c)$ : the number of threads contending for the lock. This quantifies the potential benefit of executing in transaction mode instead of mutex mode. The quantity can be measured by keeping a counter of how many threads are blocked on the lock when in mutex mode. When in transaction mode, $c$ is equal the number of threads currently executing the critical section.

- Actual contention (a): the number of times a transaction needs to try before it commits. This quantifies the contention by other threads on the actual data the critical section tries to access. The quantity is a multiplicative factor in the cost of executing the critical section in transaction mode.

- Transactional overhead (o): the slowdown factor due to transactional execution, because of the need to trap shared memory reads and writes, the need to synchronize, the need to re-validate as part of a complex contention management policy, etc. This is a multiplicative factor in the cost of transaction mode.

Thus, the cost-benefit analysis of adaptive locks is based on the inequality:

$$
a \cdot o \geq c
$$

(The two sides correspond to the overheads of each mode of execution relative to an idealized, no-contention execution. All three factors are computed separately for each adaptive lock, since the decision on which mode to execute affects all critical sections of the lock.) If this inequality holds, mutex mode execution is preferable, otherwise the benefit of transaction mode execution outweighs its cost. Note that the analysis applies and a trade-off exists even if transactional execution incurs no overhead $(o=1)$, e.g., through the use of specialized hardware.

The above cost-benefit analysis is exact and not approximate, yet approximations need to be introduced because, for instance, it is hard to measure the overhead $o$ fully accurately, factor $a$ is predictive of future executions so it needs to be estimated from past data, etc. As we describe next, factors $c$ and $a$ are computed dynamically at all 
times. Factor $o$ is also computed dynamically by sampling a subset of the executionsan approach that proved superior to off-line estimates in our measurements due to the high variance of $o$ for different applications and locks.

To see the advantage of having a complete model for cost and benefit, consider, for instance, the adaptivity approach followed by Welc et al. [10]. Their technique converts a critical section to a transactional implementation as soon as any contention is observed, i.e., as soon as $c$ is more than 1 . This completely disregards the costs of transactional execution and results in obtaining good behavior only for transactionfriendly workloads.

\section{Implementation and Optimizations}

We next describe our implementation of adaptive locks. We selectively present key components that expose the precise logic (e.g., behavior when an adaptive lock is in the process of switching modes) or reveal crucial elements for high performance.

\subsection{Compiler and Locking Mechanism}

We have implemented adaptive locks in a conservative extension of the $\mathrm{C}$ language. Our compiler is based on the CIL infrastructure [12] for extensible C compilers. A special pragma at the function level is used to supply atomic annotations: the entire body of the function is then considered to be protected by the corresponding adaptive lock. The compiler translates each function body with atomic annotations into two different object code versions: a raw version, used for mutex mode execution and incurring no further overheads, and a transactional version, where all shared memory reads and writes become TM operations for an underlying STM. We use TL2 [26], a high-performance STM library, as our back-end STM. Our implementation is freely available (see http://ix.cs.uoregon.edu/ takayuki/al/) and represents a mature open-source compiler infrastructure for STM experimentation. Other researchers can build on our compiler support for TM by modifying our CIL patterns to produce full compilers either for different TM constructs or for different back-end TM implementations. 
Our implementation of adaptive locks replaces regular lock acquisition and release with versions that perform the adaptive reasoning. We use a standard pattern for highperformance synchronization: The adaptive lock's state is packed in a memory word and we represent bit blocks as different pseudo-variables. The components of the state include the number of threads executing in transaction mode (thrdsInStmMode), whether we are currently in mutex mode (mutexMode), whether the mutex lock is held (lockHeld), and whether we are currently in the process of switching modes (transition). The next state is then computed and updated atomically with a compareand-swap (CAS) instruction. The thread spins, retrying the state update until the CAS succeeds, or until exceeding a number of tries, in which case it has to yield the CPU.

These elements are illustrated in Figure 1, which shows the state transitions of adaptive locks, as well as in in Figure 2, which contains the code for the acquire routine-the main workhorse of the lock acquisition process. This routine is called every time a thread attempts to acquire an adaptive lock. The return value indicates whether the adaptive lock was acquired in transaction mode (TRANS_MODE) or mutex mode (MUTEX_MODE). Introducing some conventions is helpful:

- The separate bit ranges of both the current state (prev) and the next state (next) are set through macros maintaining the naming convention. For instance, checking the lockHeld bit of the current state is done with the expression lockHeld (prev) whereas setting the same bit to 1 on the next state is done with the call setLockHeld (next,1). We use TRUE and FALSE for 1 and 0 , respectively, when the bit value represents a boolean.

- Atomic operations are shown in all capital letters. INC, DEC, and CAS call (directly or indirectly) atomic instructions. This will be important when we discuss performance optimizations.

- Each adaptive lock holds data for computing its adaptivity statistics. These data are not accessed directly in the code of Figure 2, with the exception of lock->thdsBlocked: a counter of threads blocked on the lock, if the lock is in mutex mode-adding thrdsInStmMode yields the $c$ factor from Section 2.2. For its adaptiv- 


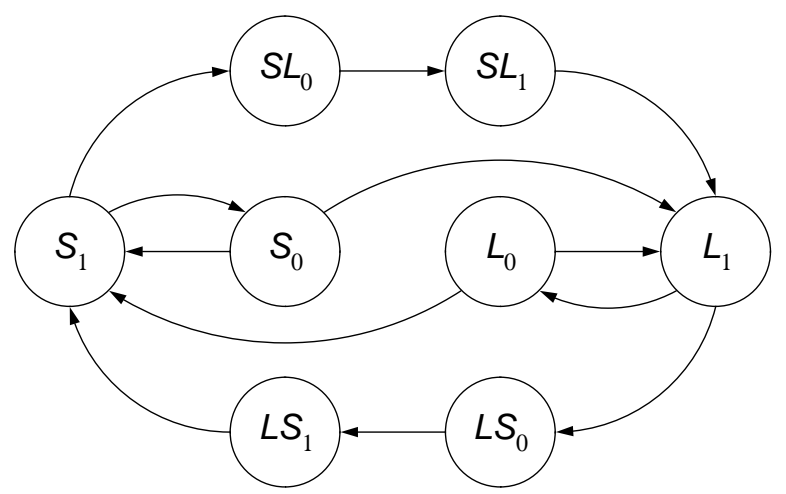

\begin{tabular}{l|l|l} 
From & To & Condition for transition \\
\hline$S_{0}$ & $S_{1}$ & Acquire, mode = STM \\
$S_{0}$ & $L_{1}$ & Acquire, mode = Lock \\
$S_{1}$ & $S_{0}$ & Release, thrdsInStmMode = 1 \\
$S_{1}$ & $S L_{0}$ & Acquire, mode = Lock \\
$L_{0}$ & $L_{1}$ & Acquire, mode = Lock \\
$L_{0}$ & $S_{1}$ & Acquire, mode = STM \\
$L_{1}$ & $L_{0}$ & Release \\
$L_{1}$ & $L S_{0}$ & Acquire, mode = STM \\
$S L_{0}$ & $S L_{1}$ & Release, thrdsInStmMode =1 \\
$L S_{0}$ & $L S_{1}$ & Release \\
$S L_{1}$ & $L_{1}$ & Acquire \\
$L S_{1}$ & $S_{1}$ & Acquire
\end{tabular}

\begin{tabular}{l|c|c|c|c|l} 
State & mutexMode & lockHeld & thrdsInStmMode & transition & Description \\
\hline$S_{0}$ & 0 & 0 & 0 & 0 & STM mode, no thread in critical region \\
$S_{1}$ & 0 & 0 & $\geq 1$ & 0 & STM mode, thread(s) in critical region \\
$L_{0}$ & 1 & 0 & 0 & 0 & Lock mode, no thread in critical region \\
$L_{1}$ & 1 & 1 & 0 & 0 & Lock mode, thread in critical region \\
$L S_{0}$ & 0 & 1 & 0 & 1 & Begin transition from lock to STM mode \\
$L S_{1}$ & 0 & 0 & 0 & 1 & Signal completed transition from lock to STM mode \\
$S L_{0}$ & 1 & 0 & $\geq 1$ & 1 & Begin transition from STM to lock mode \\
$S L_{1}$ & 1 & 0 & 0 & 1 & Signal completed transition from STM to lock mode
\end{tabular}

Figure 1: Adaptive lock state machine with explanation of states and transitions. We need two transition states in each direction, because of the Acquire/Release/Acquire handshake taking place (for ensuring progress during mode switches). The initial Acquire changes to the first transitional state, then waits for the Release operation to complete, which is signaled by the move to the second transitional state, and the Acquire then completes the transition by going to $S_{1} / L_{1}$. 


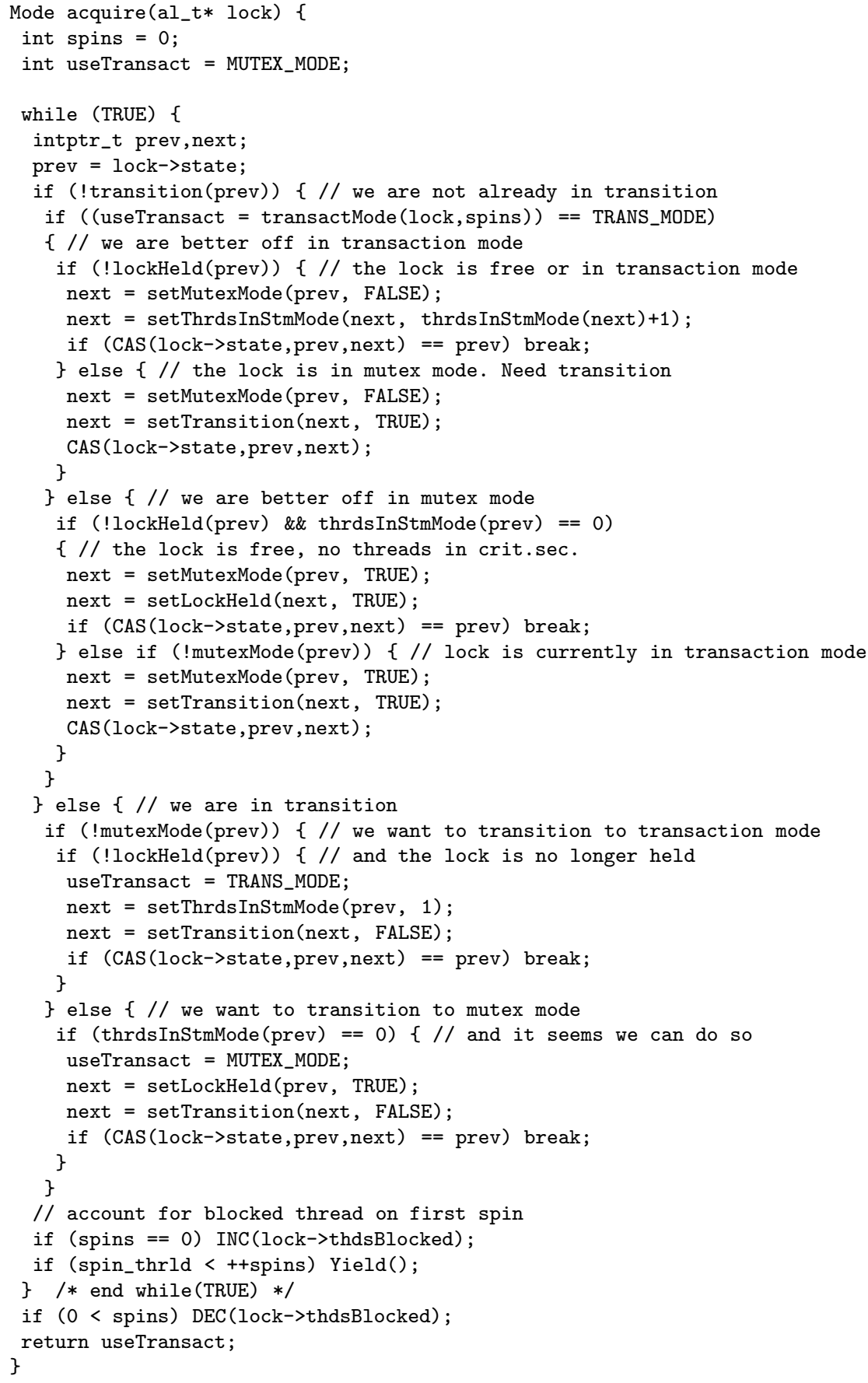

Figure 2: The main routine for adaptive lock acquisition. Returns whether the lock was acquired in mutex mode or transaction mode. 
ity logic, the acquire routine calls transactMode which implements the cost-benefit analysis of Section 2.2 and returns the estimated best mode for the adaptive lock.

We can now see precisely the behavior of adaptive locks. If the lock is not already in a state of transition from one mode to the other then the cost-benefit analysis is performed to see what is the optimal execution mode. (It is necessary for ensuring progress to choose the mode using the cost-benefit analysis only when the lock is not already in transition. Otherwise, threads that decide to acquire the adaptive lock in mutex mode might be waiting for all threads executing in transaction mode to finish. Yet new threads can keep acquiring the lock in transaction mode with no problem, thus causing the thread desiring to enter in mutex mode to wait forever.) All possibilities end with an attempt to CAS into the next state of the lock. If the CAS succeeds, in most cases we are done, unless we are switching modes, in which case the CAS will just set the state to be in-transition, and will repeat the loop until the new state is set. A failed CAS results in retrying, up to a predefined threshold of times (spin_thrld) before yielding.

When the acquire routine returns to its caller (not shown), the adaptive lock is held in the appropriate mode, and the system only needs to execute the corresponding version of the critical section (raw or transactional), per the return value. Transaction mode execution also maintains statistics for the cost-benefit analysis, namely it increments a counter for every transaction retry and commit.

\subsection{Performance Discussion}

Adaptive locks keep global statistics, necessary for computing quantities $c, a$, and $o$ of the adaptivity reasoning. Such statistics include the lock->thdsBlocked count, a count of transaction tries, and a count of transaction commits. Because these counts need to be updated by every thread's execution, they represent a global bottleneck for the performance of adaptive locks. Removing this bottleneck is crucial for performance. In some cases, even a single extra atomic instruction (e.g., a slightly less optimal implementation instead of that of Figure 2) would result in no scalability for the benchmarks we present later. 
One way in which we address this problem is by allowing small inaccuracies in our statistics gathering. The inaccuracies can only influence the performance of an adaptive lock (i.e., which mode it chooses) and not its correctness. For instance, quantity $a$ of the adaptivity reasoning (the "actual contention") is computed from counts of transaction tries and commits for the critical section. Although we make sure that these counts are not cached for long periods of time (by using volatile variables), we do not update the counts atomically. Instead, regular memory writes are performed and later instructions serve as memory barriers, forcing a shared memory update. This allows for races, including write-write races (i.e., an update being lost because a different thread overwrites it). In practical use, the sporadic inaccuracies in such statistics are not significant, especially since the counts of tries and commits are cumulative (although time-decayed).

Additionally, the transactional overhead factor, $o$, of our analysis depends on the proportion of shared memory operations (which become transactional reads and writes) in a transaction's workload. For instance, transactions that work mostly with threadlocal memory (including non-shared external resources) will not incur a heavy overhead for execution in an STM, in contrast to transactions that perform many shared memory operations. The relative mix of reads and writes also matters, depending on the specifics of the STM implementation. For instance, TL2 keeps the cost of reading shared memory low, and contains special handling for read-only transactions. For these reasons, the value of factor $o$ varies widely between applications, as well as between different critical sections of the same application.

In our implementation, we perform a dynamic measurement of $o$, using architecture-specific instruction (or cycle, when available) counters. Thus, we can estimate $o$ by measuring the execution time of a transaction, and dividing it by the execution time minus the time spent in the wrapper functions for transactional read/write memory operations (which closely approximates the time that would have been spent executing the critical section in lock mode). Getting good estimates for these times is costly, however. We found that sampling even the cheapest CPU performance counters can be prohibitive for transactions, which are typically quite brief. Furthermore, reading the values of performance counters on every TM read and write can disturb the 
behavior of the transaction, by prolonging it.

To keep our estimate of $o$ precise yet inexpensive, we apply two optimizations. First, the measurement is not performed on every transactional execution, but only in specific sampling intervals (currently every 512 calls). ${ }^{5}$ Second, we do not measure precisely how much time is spent in handling transactional reads and writes. Instead, we just keep a count of the numbers of each operation and multiply these counts by a static estimate. This is just an approximation (since the cost of reads and writes is not constant in TL2 or other STMs) but we have not found it to induce enough noise to skew our decisions.

The result of our dynamic estimation of the overhead factor is a mechanism that adapts very well to the characteristics of the application and critical section, while introducing negligible overhead, as we later show in our experiments.

\subsection{Sensitivity Discussion}

Although the cost-benefit analysis of Section 2.2 is fully general, our implementation is specialized for our back-end STM, TL2, and somewhat reflects our intended execution platform. Namely:

- The main transactional overheads of TL2 are due to read and write logging [26]. Therefore our estimate of $o$ ignores (i.e., approximates as a constant) other transactional overheads, such as the cost of acquiring locks, the cost of aborting a transaction, the cost of contention management (e.g., delaying a transaction or re-validating the read-set in order to make progress), or the variable cost of read operations due to searching the write set. These either do not apply to TL2 or are expected to be secondary factors for most workloads. Generally, to measure $o$ precisely, one needs to measure the full end-to-end cost of equivalent executions in mutex mode and in transaction mode. This is usually not feasible, as the cost is dependent on other threads, semantic equivalence is hard to establish, etc. Therefore we expect that different realizations of adaptive locks will need to employ appropriately specialized techniques for estimating $o$.

\footnotetext{
${ }^{5}$ Our implementation pre-compiles a special sampling transactional version of the critical section, which, in addition to TM operations, contains instrumentation for estimating the overhead of transactional execution.
} 
- We have not found a need to employ more scalable locking or counter techniques (e.g., avoid the bottleneck of every thread CASing the same word when the lock is in transaction mode by using a "scalable non-zero indicator", a.k.a. SNZI [27]). This may be partly because our primary execution platform (a Sun Niagara2 architecture) uses a shared L2 cache. Preliminary microbenchmarks, however, do not substantiate this theory: we found that for much higher contention/shorter transactions the performance of our technique would degrade substantially on the same architecture. We also employed a SNZI mechanism but did not observe any performance improvement for our regular benchmarks. Still, an implementation specialized for other architectures (e.g., x86) may need to employ different low-level scalability techniques.

\section{Experimental Evaluation}

To evaluate the effectiveness of adaptive locks, we performed experiments with an array of microbenchmarks (for testing boundary conditions) and macrobenchmarks. All measurements are medians of 3 runs on a Sun UltraSparc T2 (Niagara2) T5220 machine ( 8 cores with 8 threads each for a total of 64 hardware threads, at $1.2 \mathrm{GHz}$; 32 GB RAM). We used GCC 4.0.4, and our implementation of adaptive locks uses version 0.9 .4 of TL2. Our plots show the performance of our adaptive locks, compared to modes of our compiler that perform no adaptivity reasoning. (We have confirmed that the non-adaptive modes of our compiler yield virtually identical performance to plain mutexes and the STAMP STM under the base CIL compiler.)

\subsection{Microbenchmarks}

We stress-tested adaptive locks with microbenchmarks corresponding to standard mapping data structures: red-black trees, hash tables, and splay trees.

Red-black trees are the poster child benchmark for transactional memory systems. Mutex-based red-black tree solutions typically do not scale, as they use coarse-grained locking due to the very high complexity of coding a fine-grained red-black tree. TM approaches perform well because the data structure has low actual contention (different operations can access different parts of the tree without conflicts) and can benefit from increased concurrency. 
Splay trees, on the other hand, are pathologically bad for implementations that emphasize concurrency (such as TM) since every update to a part of the tree needs to change the root, which becomes a point of contention. Thus, the interesting question for splay trees is how to incur less overhead, rather than how to gain more concurrency. We use a single-lock splay tree in our experiments.

We experimented with two fixed-size hash table implementations: one with coarsegrained locking (single lock per entire table) and one with fine-grained locking (one lock per table bucket). Naturally, there is no difference in the performance of TM in the two implementations, but mutex locks perform better in the latter.

For each data structure we used a relatively high-contention workload with 50\% lookup operations, $25 \%$ inserts and $25 \%$ deletes. Each thread performs 100,000 operations total. Hash tables have 1024 buckets and the shown red-black trees have 1000 different keys. Our results are shown in Figure 3-note that these are throughput plots, so higher numbers are better. As can be seen, none of the benchmarks scales perfectly to 64 threads, largely because of the small size of the data and the resulting contention.

Adaptive locks succeed in closely tracking the performance of the better of the two component mechanisms for each benchmark. This means that adaptive locks soundly outperform either of the component mechanisms on its own. Statistically, over all microbenchmarks and all thread configurations, adaptive locks are on average $47 \%$ faster than mutexes (min: $-16 \%$, max: $433 \%$ ) and 176\% faster than transactions (min: $-26 \%$, max: $837 \%$ ). (This should only be viewed as a summary of the figure data, as the average does not map to a real-world quantity.) For red-black trees and coarse-grained hash tables, adaptive locks imitate a mutex lock for low degrees of parallelism (1-2 threads) and a TM for more threads, outperforming the mutex-based implementation. For splay trees, adaptive locks precisely match the performance of a plain mutex lock, outperforming the STM implementation. For fine-grained hash tables, adaptive locks emulate mutexes, yielding better performance than TM for few threads and identical performance for more threads. The stress-testing reveals small overheads in our adaptive locks, compared to a plain STM approach (see the difference between TM and adaptive locks in the red-black tree plot). This is due to the cost of the adaptivity logic, as discussed in Section 3.2. We observed such overheads only in stress-testing scenar- 
Red-black tree

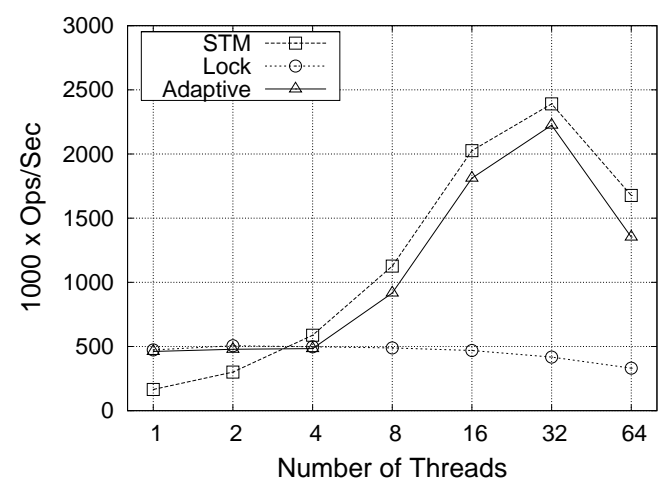

Coarse-grained Hash table

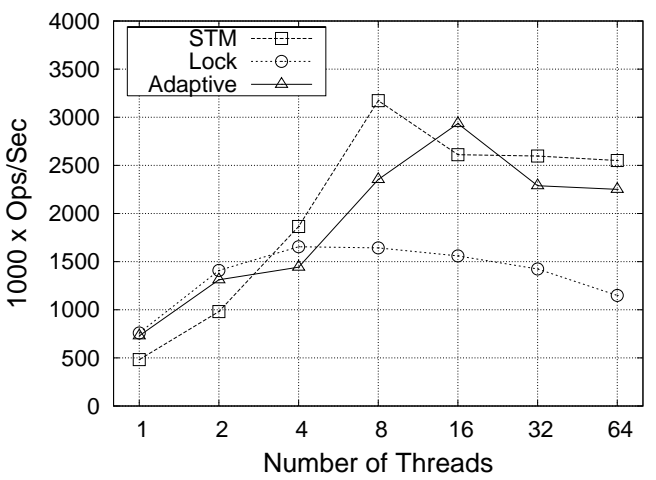

Splay tree

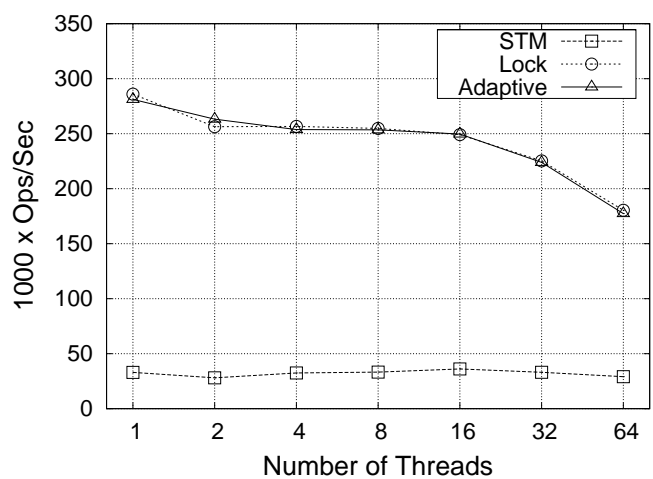

Fine-grained Hash table

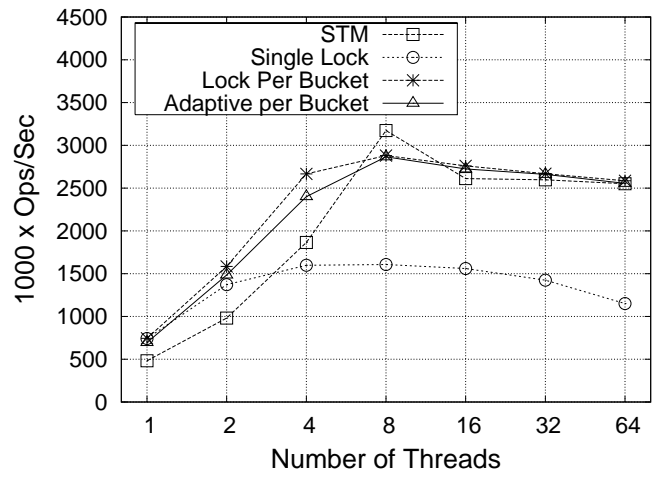

Figure 3: Microbenchmarks: Data structures with different characteristics. Higher is better. Note that the fine-grained hash table plot includes the coarse-grained mutex performance for reference. 
ios but not in more realistic settings, so we have not emphasized removing the last bit of overhead. Compared to mutex locks, our adaptive locks have no measurable overhead, as seen in the splay tree benchmark.

Sensitivity Analysis. In Section 3.3 we mentioned that our current implementation seems scalable enough for our hardware and compiler setting. Nevertheless, this does not negate the general observation that adaptive locks are a low-level mechanism whose performance depends very crucially on careful, often architecture-specific, implementation. Our code in Figure 2 offers a good example. The code has the following general structure:

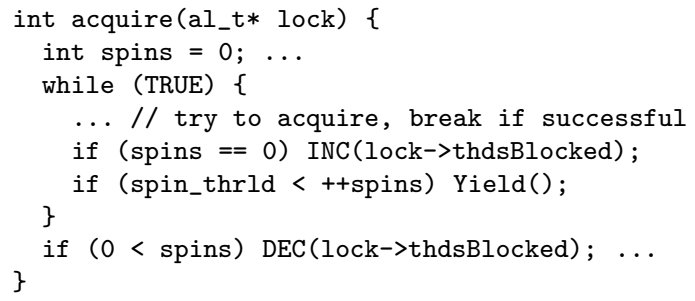

(Recall that capitalized functions denote atomic operations.) The above code only performs an INC and DEC operation after a thread starts to spin. That is, the thread is not accounted for in the thdsBlocked variable until it is delayed in acquiring the lock. This allows a slightly longer window of inaccuracy in the statistics. The alternative would be to try to update the counter as eagerly as possible: ${ }^{6}$

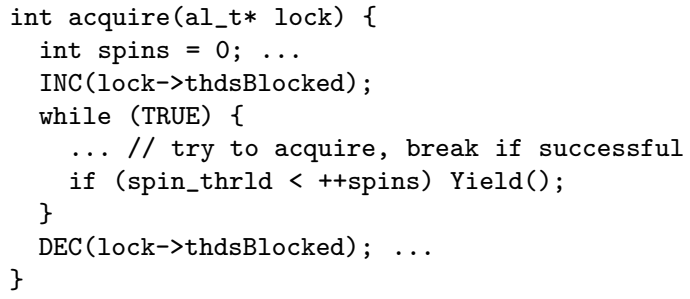

This, however, introduces two atomic operations in the critical path of an uncontested lock acquisition: there are atomic instructions before and after spinning. These

\footnotetext{
${ }^{6}$ This counter has higher accuracy requirements than the (racy) counters of transaction tries and commits, because it pertains to the current state of the lock only, instead of being cumulative (and thus tolerating more noise).
} 
can interfere unnecessarily with other threads trying to acquire the lock. Furthermore, in the case of execution in transaction mode, these instructions are a no-op for all threads: All threads do an atomic increment, attempt to acquire the lock, succeed in acquiring it in transaction mode, and immediately perform an atomic decrement.

The effect of this change is far from negligible. The performance of adaptive locks drops drastically, as the counter of spinning threads becomes a bottleneck even when in transaction mode. The result is shown in Figure 4 for the red-black tree and hash table benchmark. Comparing with Figure 3 makes evident the value of the optimized code. This also underscores the effectiveness of our adaptive locks: The challenge that our implementation meets is to provide a mechanism that is sophisticated enough to closely emulate the behavior of either mutexes or transactions, without allowing the adaptive reasoning to impose undue overhead over these low-level, performancecritical mechanisms.

Red-black tree

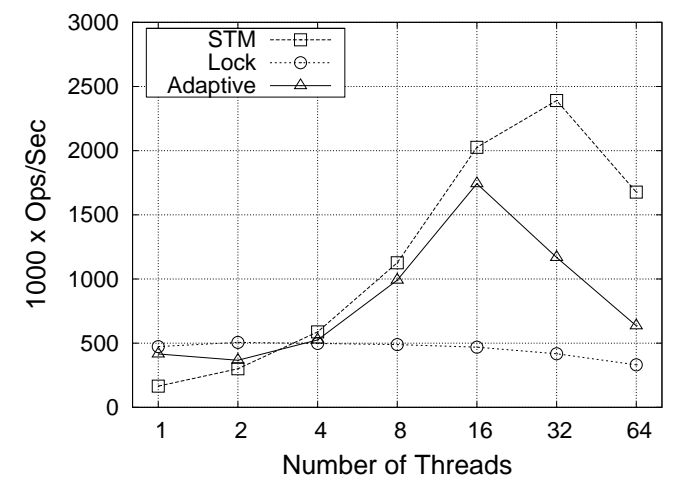

Hash table

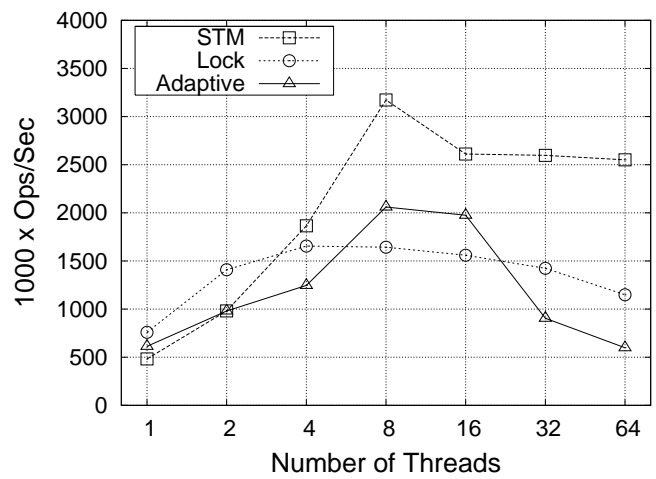

Figure 4: Microbenchmarks if more accurate accounting is performed at the expense of more atomic instructions: Adaptive locks would be unscalable in transaction mode. Compare to Figure 3. (Higher is better.)

\subsection{Macrobenchmarks}

For larger benchmarks of adaptive locks, we used the STAMP (Stanford Transactional Applications for Multi-Processing) benchmark suite [28], version 0.9.7. Subsequently, a new version of the STAMP benchmarks has been released and we ported the three new benchmark applications of STAMP 0.9.10 to also work with adaptive 
locks (while keeping the slightly different library interface of STAMP 0.9.7, which was already rewritten for adaptive locks). STAMP comprises 8 applications: bayes (a bayesian network learning program), genome (a gene sequencing program), intruder (a network intrusion detector), kmeans (an implementation of K-means clustering), labyrinth (a maze routing program), ssca2 (a graph analysis compute-intensive benchmark), vacation (a client/server travel reservation system), and yada (an implementation of Delaunay mesh refinement). All STAMP applications are written to employ a TM system explicitly. That is, the code contains explicit STM primitives (of the TL2 STM) for beginning a transaction, transactionally reading/writing a word from/to shared memory, committing a transaction, etc. As discussed in Section 3, our adaptive lock compiler supports a higher-level programming interface: all shared memory operations become implicitly transactional loads/stores when executing in transaction mode. Therefore, the STAMP applications needed careful manual modification to ensure that the output of our compiler reflects the original hand-written code, and to introduce locking annotations in the code. Our goal was to add only very coarse-grained locking, equivalent to what a programmer would be able to add with minimal effort and sophistication. Indeed, for seven out of the eight STAMP benchmarks (bayes, genome, intruder, labyrinth, ssca2, vacation, yada) we only introduced trivial locking: a single global lock for the entire application. For kmeans, 3 separate locks were introduced, with a localized code change (the critical sections for all 3 locks are in a single file and in adjacent routines).

The performance of adaptive locks for the STAMP benchmarks is illustrated in Figure 5. (Unless noted, we use the recommended options for high contention nonsimulator runs.) The graphs plot execution times, so lower is better. For a statistical summary, over all STAMP benchmarks and all thread configurations, adaptive locks are on average $103 \%$ faster than mutexes (min: $-27 \%$, max: $1021 \%$ ) and $76 \%$ faster than TM (min: $-35 \%$, max: $660 \%$ ).

Adaptive locks track very closely, and even outperform the better of the two component mechanisms over all applications. We should note that we have low confidence in the results for yada: we have already fixed a number of performance and correctness bugs in the application (independent of adaptive locks) and the measure- 
bayes

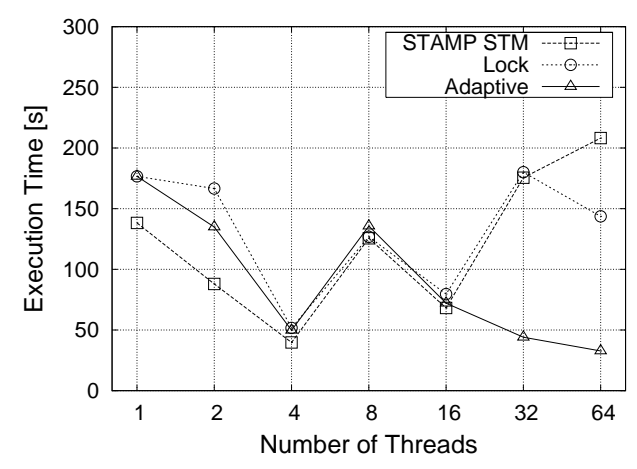

intruder

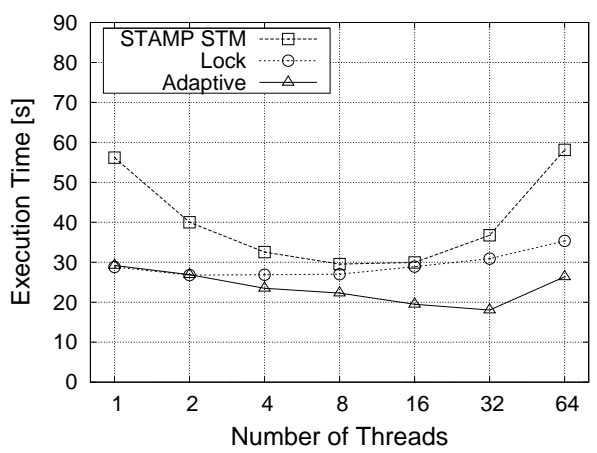

labyrinth

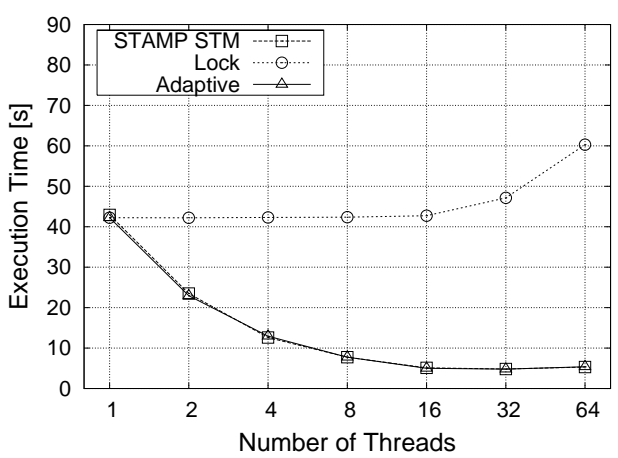

vacation

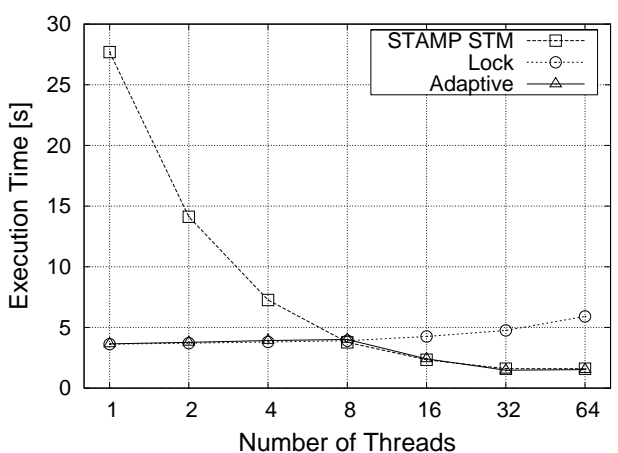

genome

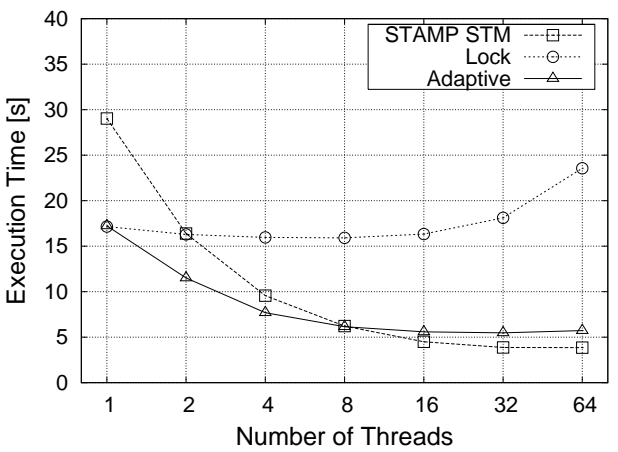

kmeans

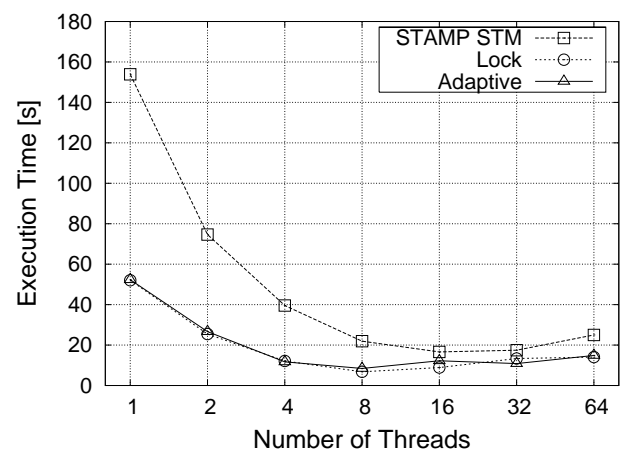

ssca 2
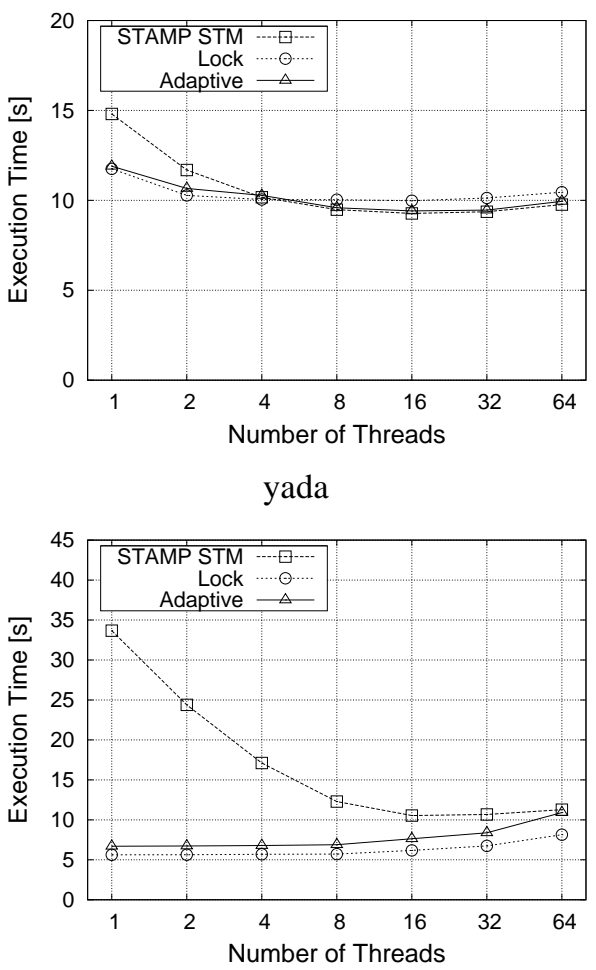

Figure 5: STAMP benchmarks, for varying numbers of threads. Lower is better. 
ments shown were taken after performing an overly conservative, STM-disadvantaging rewrite (turned many local operations into transactional ones) to avoid crashes. (More study and a deeper understanding of the yada semantics will undoubtedly address this problem.) For labyrinth, adaptive locks imitate TM behavior and vastly outperform mutex locks for all thread configurations. For kmeans, adaptive locks imitate mutexes and outperform the TL2 STM for all thread configurations. The behavior of bayes is unstable by its nature (the STAMP documentation reads "for multithreaded runs, the running time can vary depending on the insertion order of edges", hence performance is dependent on the scheduling order of threads) but adaptive locks consistently perform well for 4 or more threads. More interesting behavior can be seen for genome, ssca2, and vacation, where adaptive locks emulate mutexes for best performance with a low number of threads, while executing in transactional mode and perfectly matching or beating the performance of plain TL2 for higher numbers of threads. Occasionally, adaptivity is profitable even in the course of the same execution. For instance, for most of the intruder data points, as well as for genome in a 2-4 thread configuration, the adaptive lock version of the program profitably switches modes during execution, outperforming both mutexes and transactions alone.

Overall, the performance of adaptive locks for STAMP benchmarks validates the approach very well. Our use of only coarse-grained adaptive locking illustrates the intended usage mode of the mechanism. Adaptive locks simplify the multi-threaded programming model, by allowing the programmer to write coarse-grained annotations and achieve easy multi-threaded correctness. The convenience comes without sacrificing concurrent performance: The adaptivity mechanism can detect when coarse-grained locking is too conservative and recover concurrency (as if using fine-grained locks) by executing in transaction mode.

Sensitivity Analysis. Adaptive locks may be most useful in settings where transactional mechanisms are pessimal. It is hard or impossible to statically discern when such cases may arise. For instance, a single transactional implementation of a data structure may be included in a program and tested to perform well on its expected platform and program inputs. When, however, the platform or program inputs vary (e.g., be- 
cause other cores on the machine are occupied, or because the input causes contention or long-running transactions) performance can be severely sub-optimal. As discussed earlier, transactional memory mechanisms in software can suffer very high overheads. Adaptive locks are an automatic way to protect against such variation in setting. Our benchmarking revealed such cases. For example, Figure 6 shows the performance of the STAMP vacation benchmark for a very high contention (the highest of the "recommended") setting and a large size (outside the range recommended for "simulator" execution but still smaller than the non-simulator range). The benchmark consists of a number of agents performing queries on a database residing in an in-memory red-black tree. The vast majority of the program's running time is spent within several heavyweight (long) critical sections. The STM's memory access overhead is crippling in this scenario. The benchmark is orders of magnitude faster with locks than it is with transactional memory. At the same time, this setting makes concurrency yield no benefit for the benchmark. This is not the intended deployment domain for vacation, but it is a perfectly valid input and within the STAMP input recommended range. The large discrepancy in performance, together with the precise imitation of mutex locks, showcase why adaptive locks can be highly desirable compared to a hard-coded implementation choice.

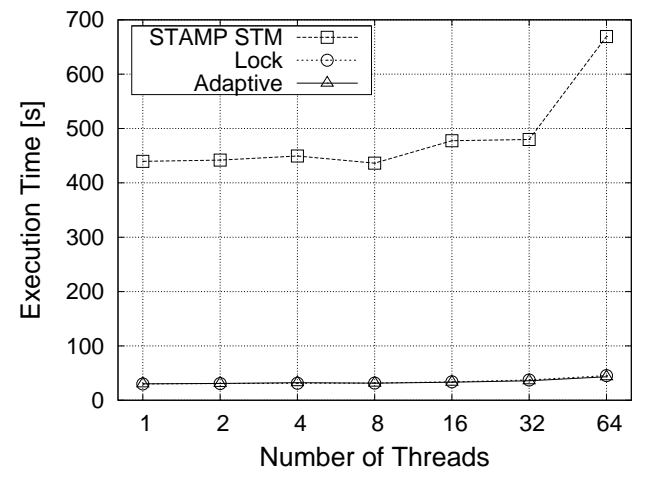

Figure 6: Vacation benchmark for highest contention and large input. STM performs very badly, but adaptive locks ignore it. (Lower is better.) 


\section{Semantic Discussion}

It is interesting to note that the transaction and mutex modes of adaptive locks are not always equivalent. The topic of the semantic differences between locks and transactions has been covered in significant detail in previous literature $[11,14,16-$ $18,29]$, and we next discuss how the differences affect adaptive locks.

Although both transactions and mutex locks enforce isolation, mutex locks also have barrier semantics for both lock acquisition and release, ensuring that all preceding memory operations are visible to all threads. This can produce surprising results if the programmer uses adaptive locks with the expectation of getting the exact behavior of mutex locks. The cases of interest can be broadly classified as privatization/publication patterns $[14,29,30]$ and lock nesting.

We should note that the our adaptivity ideas are orthogonal to such semantic differences. For instance, adaptive locks can employ a transactional memory system enforcing strong atomicity $[14,31]$ or single-global-lock semantics [17], which would avoid all semantic differences with privatization/publication patterns. Nevertheless, implementations of adaptive locks may opt to emphasize performance at the expense of mutex-like semantics, therefore the discussion of this section is highly pertinent. In particular, our current implementation of adaptive locks uses a TM that does exhibit semantic differences from mutex locks.

\subsection{Privatization/Publication patterns}

Consider the following privatization example, adapted from [14]. (The case of publication [29] is analogous and our statements apply. In fact, it is even harder for an efficient STM to be publication-safe.) 


\begin{tabular}{l|c}
\multicolumn{1}{c}{ Thread 1 } & Thread 2 \\
\hline Item *item; & atomic (listlock) \{ \\
atomic (listlock) \{ & if (!isEmpty(list)) \{ \\
item = & getFirst(list); \\
removeFirst(list); & item->val1++; \\
int r1 = item->val1; & item->val2++; \\
int r2 = item->val2; & \} \\
// Can r1 != r2 ? & \} \\
\hline
\end{tabular}

Assume that the program wants to maintain the invariant item->val1 == item->val2 throughout the execution. If the critical sections are executed in mutex mode, the above code is correctly synchronized, with no race conditions, and the invariant is kept. The two accesses to the item values in thread 1 are safe because the item has been removed from the shared data structure ("privatized") and therefore cannot be accessed by other threads - there is no way to observe intermediate states with a changed val1 but not val2. This is not, however, necessarily the case when the critical sections are executed in transaction mode. For instance, consider our current implementation of adaptive locks, which uses TL2 [26] as its underlying TM system. TL2 uses a "deferred update" approach, where writes to memory are stored in a log. A transaction commits by first locking all the memory words written by the transaction, then validating all memory words read (by checking their "version numbers") and finally copying the updated values from the log to the written words in shared memory. In this example, the two transactions do not write to the same words. Therefore, transaction 2 can commit "first" (i.e., validate its read of the first data structure item before transaction 1 updates it) yet, while it writes to shared memory the changes to item->val1 and item->val2, transaction 1 can commit, removing item from the data structure while it is being updated.

One way to view the problem is that TL2 guarantees the serializability of transactions only for direct read-write and write-write conflicts, and not for indirect conflicts. In this example, the transactional system has no way of knowing that the writes to 
item->val1 and item->val2 can conflict with the read actions of Thread 1 , since these are outside all transactions. In other cases, such conflicts would be races even in the mutex mode of execution of an adaptive lock. Nevertheless, privatization is a special case, as it makes the data structure element invisible to any other thread.

This observation leads to a simple (though perhaps too strict) criterion for the equivalence of mutex mode and transaction mode execution of adaptive locks: For each shared memory location there should be a lock, such that every access to the shared memory location occurs with the lock held. This is a form of a static-separation criterion for partitioning shared data according to their expected accesses. Indeed, this is the standard lockset [32] well-formedness criterion for multi-threaded programs. The lockset heuristic has been used (in its pure form or with various refinements) as the basis of some of the best known race detectors and multi-threaded correctness checkers [32-35]. We can check that a program respects the lockset correctness condition using any of these static or dynamic analyses. Note that this condition disallows our privatization example. If the program does respect the lockset criterion, then all possible (low-level) races are prevented by the TM system, as shared data are always accessed while holding an adaptive lock (i.e., inside a transaction, when in transaction mode). This guarantees the safety of transactional execution if mutex mode execution is safe. Still, as explained in the next section, this is not a full correctness guarantee.

Other, more permissive, programming models for transactions with weak atomicity have been recently proposed and can be similarly adapted to our setting. For instance, dynamic separation [20] requires that data be explicitly "released" at run-time, when they go from being accessed inside transactions to being accessed outside transactions. This can be again used to make the transactional memory mechanism handle privatization and publication correctly, without imposing undue overhead to regular transaction execution.

\subsection{Lock Nesting}

We view nesting as the complementary problem of privatization (and weaker atomicity models, more generally) in the semantic differences between transactions and locks. Nesting concerns progress properties while atomicity models concern safety. 
Nesting causes problems when programmers expect that the effects of a shared memory operation become accessible to other threads at the point of a lock-release. Indeed, most concurrent memory models guarantee that a lock-release operation acts as a memory barrier, resulting in the flushing of write buffers. When transactions are nested, however, their results do not become visible until the outer transaction commits-this is the standard closed-nested semantics of transactions. In this case, implementing the outer critical section as a transaction is incorrect. To see the problem, consider the following example. Function barrier implements a simple barrier by spinning until all threads reach the same point.

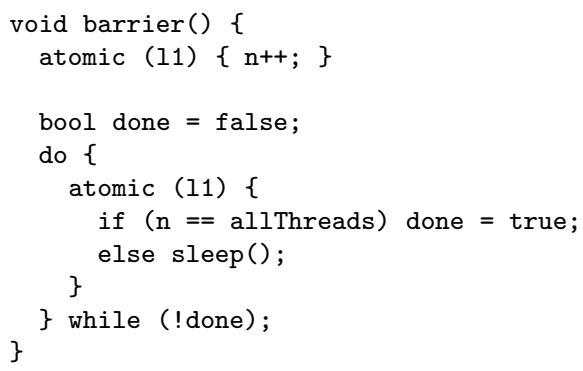

The problem begins when the barrier routine happens to be used inside a different critical section, possibly protecting completely distinct data.

atomic (12) $\{\ldots$ barrier ()$; \ldots\}$

If this code executes in mutex mode, the barrier is correct. If, however, the code executes in transaction mode, then the execution of the entire outer transaction (on adaptive lock 12) happens in an all-or-nothing way. That is, the increment $n++$ can never be visible to other threads until the outer transaction commits, which will never happen, as the thread needs to see the $\mathrm{n++}$ actions of all other threads prior to exiting the barrier.

This semantic difference between mutex and transaction mode is a much less serious problem than that of privatization, as it only arises for low-level mechanisms that rely on noticing updates to shared data and reacting to them while holding locks. Doing so, however, requires repeated sampling of the shared data-i.e., spinning. In practice, most programs will implement shared-data communication using some highlevel mechanism, such as condition variables. Past studies [36, 37] have also found 
lock nesting to be rare in existing multi-threaded applications (although other studies disagree for the domain of kernel synchronization [38]). Despite the low practical interest, however, it is worthwhile to discuss this case for completeness: It is an interesting question whether we can ensure the full equivalence of mutex and transaction modes, even for low-level coding patterns.

Note that the lockset criterion of Section 5.1 does not protect us against this semantic issue. For instance, the barrier example does respect the lockset heuristic, as every shared memory word is accessed with 11 held. Nevertheless, there is a simple additional condition that prevents such problems: If the set of held locks (i.e., all surrounding locks) is the same for every thread that tries to enter a critical section protected by lock $l$, then transaction mode and mutex mode execution are equivalent. This condition does not require a separate analysis of the program. It can be checked at run-time, when a thread acquires adaptive lock $l$ and the adaptivity mechanism decides to execute the critical section in transaction mode. In this case, the set of already held locks is compared against the set of held locks by other threads that also hold $l$. If the two sets differ, then the original critical section needs to be aborted and restarted in mutex mode. Our current adaptive locks implementation does not support this mechanism for reasons of engineering and because of its questionable practical impact.

Why is this condition sufficient for preventing semantics differences, however? The reason is that for the semantic difference to become apparent, a thread needs to be able to observe the effects of an inner critical section, without needing to hold the same outer lock (or else it would be prevented from entering the outer lock even in mutex mode). Consider our barrier example. The essence of the problem is that the barrier routine can be called while sometimes holding lock 12 and sometimes not. If all threads consistently called barrier while holding 12, then the result would suffer from the lack-of-progress error, but the error would also exist in mutex mode. Only when a thread is allowed to call barrier without holding 12 is the mutex mode execution correct, while the transaction mode one is incorrect.

To summarize, we have shown that although the semantics of mutex mode and transaction mode are not identical, the difference is confined to limited cases. The most important one in practice is the case of privatization and publication, which can 
be detected using standard multi-threaded correctness analyses.

\section{Related Work}

We discussed directly related work throughout the previous sections. Here we outline some work that is less directly related, yet offers context for our work, or explores closely related directions in different settings.

Transactions originated in the databases research literature [39] before they transitioned to general-purpose programming in the form of transactional memory [40]. Although the principles are similar, the challenges in the two domains are quite distinct. For instance, TM has to allow for arbitrary memory accesses and, thus, cannot generally predict all locks that need to be acquired. Furthermore, the granularity of access is finer in TM, creating very different trade-offs for high-performance implementations.

In the database world, our adaptive locks might be described as a mechanism adapting between optimistic concurrency control and pessimistic concurrency control. The term "optimistic" refers to allowing transactions to proceed in the hope that they will not conflict, while installing mechanisms to detect such conflicts. The term "pessimistic" refers to acquiring locks up front, so that any transactions that have the possibility of conflict end up serializing. Database researchers have explored combinations of optimistic and pessimistic concurrency control, and so have researchers in automatic parallelization [21, 41]. The options are sometimes said to be akin to "apologizing versus asking permission" [42]. The mutex mode of our adaptive locks is an ultra-pessimistic mechanism, as it forces all transactions to "ask permission" up front. Receiving permission means that the transaction can proceed and is guaranteed to not roll back: it has "reserved" the right to perform its memory operations.

The PhTM [43] system is related to our work in that it describes a mechanism for dynamically switching synchronization mechanisms. Nevertheless, our work advances the PhTM ideas in several ways. First, PhTM introduces only a single global lock instead of individual locks. Second, although the PhTM "Sequential-Noabort" mode supports switching to lock-based execution, the PhTM prototype does not support such switching. In fact, the PhTM authors speculate, "we can likely improve performance in most cases by monitoring progress of transactions, commit/abort rates, status of 
transactions with respect to the current mode, etc." and conclude that "[f]uture work includes ... mechanisms for deciding when to switch to what mode." Our work directly addresses these topics. Other researchers have recently also proposed mechanisms for replacing locks with software transactions [44], again without a cost-benefit adaptivity model to guide a run-time mode switch.

Several other recent research projects have either alternated between pessimistic and optimistic TM implementations or provided support for irrevocable/single-mutexmode transactions [45, 46]. Additionally, the issue of contention management [47-49] is closely related to adaptive mode switching. A contention manager decides on a policy for favoring transactions in the case of contention, possibly radically switching the performance characteristics of the system. A close relative of adaptive locks in this space is the work of $\mathrm{Ni}$ et al. [48], which describes a real-world implementation of transactional constructs for $\mathrm{C}$ and $\mathrm{C}++$. Their approach includes multiple mechanisms that are related to switching implementation policy, including transactions that can perform irrevocable operations, transactions that put all other transactions on hold, and switching a transaction between optimistic and pessimistic concurrency control. Nevertheless, neither the $\mathrm{Ni}$ et al. work, nor other work on irrevocable transactions or contention management offer a clear cost-benefit model for dynamically deciding concurrency vs. exclusion for best performance. Specifically, none of the above work seems to collect statistics on nominal contention per lock, or on transactional execution overhead, although taking the number of transaction (re-)tries into account is common.

Our exploration of adaptive locks is in the context of a pure software implementation. An important trend is to provide hardware support for TM [50-61]. With hardware support, the performance trade-offs change-e.g., the transactional overhead of loads and stores may be virtually eliminated. Yet the idea of adaptive locks should be quite applicable to hardware TMs: even with no overhead for TM execution, it will be beneficial to adaptively detect when transactions have high actual contention and mutual exclusion would be profitable. (In practice it is unlikely that TM execution will incur truly no overhead, i.e., that the factor $o$ will be 1. Failed transactions can generate bus traffic that will slow down successful transactions. Additionally, the system may prefer to delay transactions and retry a memory operation, rather than aborting, possi- 
bly also for energy reasons. Overall, it is likely that all three factors $o, a$, and $c$ of our cost-benefit analysis will play a role, even in hardware implementations.) Furthermore, most hardware support for TM employs a hybrid software-hardware approach-e.g., transactions that access a lot of shared data need to be implemented in software, making our approach perfectly applicable. Finally, many of the adaptivity ideas of this paper (e.g., keeping track of the number of transaction tries or blocked threads and deciding the execution mode based on those) can be employed in hardware mechanisms such as speculative lock elision [9] or optimistic thread concurrency [61].

Dice et al. [62] present a survey of tests on the Rock multicore processor's hardware transactional memory feature. The feature is described as a best-effort HTM. It can fail for any number of reasons and, when it does, it relies on software to handle the aborted transaction. In addition, a register is set to detail the cause of the failure. Causes include coherence violations, branch mispredictions or the simple filling of the hardware buffer that holds changes prior to commit. The experiments handle the aborts by either retrying, backing off, switching to an STM or switching to a lock. A simple notion of adaptivity is employed, since the switches only occur after a certain amount of aborts and, in some scenarios, different causes for aborts are weighted differently.

The TxLinux work [38] provides synchronization primitives to allow transactionand mutex-based synchronization to co-exist. The mechanism automatically detects $\mathrm{I} / \mathrm{O}$ and restarts the running transaction in mutex mode. TxLinux is implemented using special primitives offered by its HTM that adopts eager conflict detection and in-place updates, and some special features of its processor to support I/O detection. No adaptivity between transaction and mutex is explored for performance.

Volos et al. [63] introduce xCalls: wrapper functions around some system calls that provide transaction-friendly semantics. xCalls work by deferring side effects until commit or executing immediately and reverting at commit time. xCalls do not have wrappers for system calls that cannot be dealt with on these terms. A number of the calls use user-level OS locks on system resources to prevent other threads from seeing changes from in-flight transactions. It would be interesting to integrate xCalls with adaptive locks, in order to expand the space of code that can execute in transaction mode (by including also code that performs some I/O calls). Nevertheless, the integra- 
tion would require significant work, because of the possibility of "undone" effects of past system calls existing when we switch from transaction mode to lock mode. For instance, on a pipe read call, xCalls keeps a buffer of destroyed data, to be used in case of a transaction abort and retry. Switching to mutex mode would result in calling the unwrapped system call and missing these data.

\section{Conclusions}

We presented the idea of adaptive locks as a concurrency control construct for multi-threaded programming. A major contribution of our work is in identifying the statistics needed for an effective cost-benefit adaptivity analysis and in developing mechanisms for maintaining such statistics highly efficiently. Overall, we believe that our work establishes adaptive locks as an excellent candidate for inclusion in industrialstrength systems.

The efficiency of our adaptivity approach suggests several other future applications. One promising possibility is adaptive switching between a pessimistic and an optimistic TM implementation-i.e., rolling back on deadlock vs. rolling back on actual contention. Although both pessimistic and optimistic TM approaches perform best when there is no actual contention on the data, pessimistic TM approaches reduce the probability of roll-back at the expense of some thread waiting for locks to become available. Adaptivity can help determine when this trade-off is profitable. Another potential application of adaptivity is in changing the locking granularity of a TM system: an adaptive TM can start with associating lock words (used internally by the TM implementation) at a coarse granularity. Then, as nominal contention is encountered, the system can switch to a finer granularity, and possibly back, if the overhead of fine-grained locking appears heavy and unwarranted. Such granularity switching is applicable to practically all TM systems we are aware of, both pessimistic and optimistic. Our adaptive locks can be seen as an extreme case of both granularity switching and pessimistic-optimistic switching at the same time.

Generally, we believe that the idea of adaptive concurrency control holds significant promise and that adaptive locks, as analyzed in this paper, are an excellent representative of the possibilities. 


\section{Acknowledgments.}

This work was supported by Sun Microsystems via an equipment grant, by the National Science Foundation under grants CCF-0917774 and CCF-0934631, as well as by LogicBlox Inc. We would like to thank Doug Lea for his support and access to equipment, Michal Young for several insightful conversations, as well as the anonymous TRANSACT'09, PACT'09, and JPDC reviewers for many comments that helped improve the paper.

\section{References}

[1] M. I. Gordon, W. Thies, S. Amarasinghe, Exploiting coarse-grained task, data, and pipeline parallelism in stream programs, in: ASPLOS-XII: Proceedings of the 12th international conference on Architectural support for programming languages and operating systems, ACM, 2006, pp. 151-162.

[2] J. Armstrong, R. Virding, C. Wikström, M. Williams, Concurrent Programming in ERLANG, second edition, Prentice Hall, 1996.

[3] Y. Smaragdakis, A. Kay, R. Behrends, M. Young, Transactions with isolation and cooperation, in: ACM Symposium on Object Oriented Programming: Systems, Languages, and Applications (OOPSLA), ACM Press, 2007.

[4] C. Blundell, E. C. Lewis, M. M. K. Martin, Unrestricted transactional memory: Supporting I/O and system calls within transactions, Technical Report CIS-06-09, Department of Computer and Information Science, University of Pennsylvania (Apr 2006).

[5] B. D. Carlstrom, J. Chung, H. Chafi, A. McDonald, C. Cao Minh, L. Hammond, C. Kozyrakis, K. and Olukotun, Transactional execution of java programs, in: OOPSLA 2005 Workshop on Synchronization and Concurrency in ObjectOriented Languages (SCOOL), 2005.

[6] T. Harris, Exceptions and side-effects in atomic blocks, Science of Computer Programming 58 (3) (2005) 325-343. doi:http://dx.doi.org/10.1016/j.scico.2005.03.005. 
[7] C. Cascaval, C. Blundell, M. Michael, H. W. Cain, P. Wu, S. Chiras, S. Chatterjee, Software transactional memory: why is it only a research toy?, Commun. ACM 51 (11) (2008) 40-46. doi:http://doi.acm.org/10.1145/1400214.1400228.

[8] C. Boyapati, M. Rinard, A parameterized type system for race-free java programs, in: OOPSLA '01: Proceedings of the 16th ACM SIGPLAN conference on Object-oriented programming, systems, languages, and applications, ACM, New York, NY, USA, 2001, pp. 56-69. doi:http://doi.acm.org/10.1145/504282.504287.

[9] R. Rajwar, J. Goodman, Speculative lock elision: Enabling highly concurrent multithreaded execution, 34th International Symposium on Microarchitecture, December $00 \quad$ (2001) 294. doi:http://doi.ieeecomputersociety.org/10.1109/MICRO.2001.991127.

[10] A. Welc, A. L. Hosking, S. Jagannathan, Transparently reconciling transactions with locking for java synchronization, in: European Conference on ObjectOriented Programming, 2006, pp. 148-173.

[11] Y. Smaragdakis, A. Kay, R. Behrends, M. Young, General and efficient locking without blocking, in: ACM SIGPLAN Workshop on Memory Systems Performance and Correctness (MSPC), 2008.

[12] G. C. Necula, S. McPeak, S. P. Rahul, W. Weimer, CIL: Intermediate language and tools for analysis and transformation of C programs, in: CC '02: Proc. 11th Int. Conf. on Compiler Construction, Springer-Verlag, London, UK, 2002, pp. 213-228.

[13] T. Harris, K. Fraser, Language support for lightweight transactions, in: OOPSLA '03: Proc. 18th conf. on Object-oriented Programing, Systems, Languages, and Applications, ACM Press, Anaheim, CA, 2003, pp. 388-402. doi:http://doi.acm.org/10.1145/949305.949340.

[14] T. Shpeisman, V. Menon, A.-R. Adl-Tabatabai, S. Balensiefer, D. Grossman, R. L. Hudson, K. F. Moore, B. Saha, Enforcing isolation and ordering in STM, in: PLDI 
'07: Proceedings of the 2007 ACM SIGPLAN Conference on Programming Language Design Implementation, ACM Press, New York, NY, USA, 2007, pp. 7888. doi:http://doi.acm.org/10.1145/1250734.1250744.

[15] T. Harris, M. Herlihy, S. Marlow, S. Peyton-Jones, Composable memory transactions, in: Proc. Symposium on Principles and Practice of Parallel Programming, 2005.

[16] C. Blundell, E. C. Lewis, M. M. Martin, Subtleties of transactional memory atomicity semantics, IEEE Comput. Archit. Lett. 5 (2) (2006) 17. doi:http://dx.doi.org/10.1109/L-CA.2006.18.

[17] V. Menon, S. Balensiefer, T. Shpeisman, A.-R. Adl-Tabatabai, R. L. Hudson, B. Saha, A. Welc, Single global lock semantics in a weakly atomic STM, in: 3rd workshop of Transactional Computing (TRANSACT), 2008.

[18] D. Grossman, J. Manson, W. Pugh, What do high-level memory models mean for transactions?, in: ACM SIGPLAN Workshop on Memory Systems Performance and Correctness (MSPC), ACM, New York, NY, USA, 2006, pp. 62-69. doi:http://doi.acm.org/10.1145/1178597.1178609.

[19] M. Abadi, A. Birrell, T. Harris, M. Isard, Semantics of transactional memory and automatic mutual exclusion, in: G. C. Necula, P. Wadler (Eds.), POPL, ACM, 2008, pp. 63-74.

[20] M. Abadi, A. Birrell, T. Harris, J. Hsieh, M. Isard, Implementation and use of transactional memory with dynamic separation, in: Compiler Construction, 2009.

[21] M. C. Rinard, Effective fine-grain synchronization for automatically parallelized programs using optimistic synchronization primitives, ACM Transactions on Computer Systems 17 (4) (1999) 337-371.

URL citeseer . ist.psu .edu/rinard99effective.html

[22] N. Shavit, D. Touitou, Software transactional memory, in: PODC, 1995, pp. 204213. 
[23] S. Cherem, T. Chilimbi, S. Gulwani, Inferring locks for atomic sections, in: SIGPLAN Conference on Programming Language Design and Implementation (PLDI), ACM, New York, NY, USA, 2008, pp. 304-315. doi:http://doi.acm.org/10.1145/1375581.1375619.

[24] B. McCloskey, F. Zhou, D. Gay, E. Brewer, Autolocker: Synchronization inference for atomic sections, in: POPL '06: Proc. 33rd symposium on Principles of Programming Languages, ACM Press, 2006, pp. 346-358. doi:http://doi.acm.org/10.1145/1111037.1111068.

[25] M. Hicks, J. S. Foster, P. Prattikakis, Lock inference for atomic sections, in: Proceedings of the First ACM SIGPLAN Workshop on Languages, Compilers, and Hardware Support for Transactional Computing, 2006.

[26] D. Dice, O. Shalev, N. Shavit, Transactional locking II, in: S. Dolev (Ed.), Distributed Computing, 20th International Symposium (DISC), Vol. 4167 of Lecture Notes in Computer Science, Springer, 2006.

[27] F. Ellen, Y. Lev, V. Luchangco, M. Moir, Snzi: scalable nonzero indicators, in: PODC '07: Proceedings of the twenty-sixth annual ACM symposium on Principles of distributed computing, ACM, New York, NY, USA, 2007, pp. 13-22. doi:http://doi.acm.org/10.1145/1281100.1281106.

[28] C. C. Minh, J. Chung, C. Kozyrakis, K. Olukotun, STAMP: Stanford transactional applications for multi-processing, in: IISWC '08: Proc. IEEE International Symposium on Workload Characterization, 2008.

[29] V. Menon, S. Balensiefer, T. Shpeisman, A.-R. Adl-Tabatabai, R. L. Hudson, B. Saha, A. Welc, Practical weak-atomicity semantics for Java STM, in: SPAA '08: Proceedings of the twentieth annual symposium on Parallelism in algorithms and architectures, ACM, New York, NY, USA, 2008, pp. 314-325. doi:http://doi.acm.org/10.1145/1378533.1378588.

[30] J. R. Larus, R. Rajwar, Transactional Memory, Morgan \& Claypool, 2007. 
[31] F. T. Schneider, V. Menon, T. Shpeisman, A.-R. Adl-Tabatabai, Dynamic optimization for efficient strong atomicity, in: OOPSLA '08: Proceedings of the 23rd ACM SIGPLAN conference on Object-oriented programming systems languages and applications, ACM, New York, NY, USA, 2008, pp. 181-194. doi:http://doi.acm.org/10.1145/1449764.1449779.

[32] S. Savage, M. Burrows, G. Nelson, P. Sobalvarro, T. Anderson, Eraser: a dynamic data race detector for multi-threaded programs, in: SOSP '97: Proc. 16th Symposium on Operating Systems Principles, ACM, New York, NY, USA, 1997, pp. 27-37. doi:http://doi.acm.org/10.1145/268998.266641.

[33] C. Flanagan, S. N. Freund, Atomizer: A dynamic atomicity checker for multithreaded programs, Sci. Comput. Program. 71 (2) (2008) 89-109. doi:http://dx.doi.org/10.1016/j.scico.2007.12.001.

[34] B. Krena, Z. Letko, R. Tzoref, S. Ur, T. Vojnar, Healing data races on-the-fly, in: PADTAD '07: Proceedings of the 2007 ACM workshop on Parallel and distributed systems: testing and debugging, ACM, New York, NY, USA, 2007, pp. 54-64. doi:http://doi.acm.org/10.1145/1273647.1273658.

[35] C. von Praun, T. R. Gross, Object race detection, in: OOPSLA '01: Proceedings of the 16th ACM SIGPLAN conference on Object oriented programming, systems, languages, and applications, ACM, New York, NY, USA, 2001, pp. 70-82. doi:http://doi.acm.org/10.1145/504282.504288.

[36] B. B. Brandenburg, J. H. Anderson, Feather-trace: A light-weight event tracing toolkit, Proceedings of the Third International Workshop on Operating Systems Platforms for Embedded Real-Time Applications (2007) 20-27.

[37] D. F. Bacon, R. Konuru, C. Murthy, M. Serrano, Thin locks: featherweight synchronization for java, in: PLDI '98: Proceedings of the ACM SIGPLAN 1998 conference on Programming language design and implementation, ACM, New York, NY, USA, 1998, pp. 258-268. doi:http://doi.acm.org/10.1145/277650.277734. 
[38] C. J. Rossbach, O. S. Hofmann, D. E. Porter, H. E. Ramadan, A. Bhandari, E. Witchel, Txlinux: using and managing hardware transactional memory in an operating system, in: SOSP '07: Proceedings of twenty-first ACM SIGOPS Symposium on Operating Systems Principles, ACM, New York, NY, USA, 2007, pp. 87-102. doi:http://doi.acm.org/10.1145/1294261.1294271.

[39] J. Gray, A. Reuter, Transaction Processing: Concepts and Techniques, Morgan Kaufmann Publishers Inc., San Francisco, CA, USA, 1992.

[40] M. Herlihy, J. E. B. Moss, Transactional memory: Architectural support for lockfree data structures, in: Proceedings of the 20th Annual International Symposium on Computer Architecture, 1993, pp. 289-300.

[41] P. C. Diniz, M. C. Rinard, Dynamic feedback: An effective technique for adaptive computing, in: SIGPLAN Conference on Programming Language Design and Implementation (PLDI), 1997, pp. 71-84.

URL citeseer.ist.psu.edu/diniz97dynamic.html

[42] M. Herlihy, Apologizing versus asking permission: Optimistic concurrency control for abstract data types, ACM Trans. Database Syst. 15 (1) (1990) 96-124.

[43] Y. Lev, M. Moir, D. Nussbaum, PhTM: Phased transactional memory, in: TRANSACT 07: Proceedings of the second ACM SIGPLAN Workshop on Languages, Compilers, and Hardware Support for Transactional Computing, 2007.

[44] A. Roy, S. Hand, T. Harris, A runtime system for software lock elision, in: Eurosys, 2009.

[45] A. Welc, B. Saha, A.-R. Adl-Tabatabai, Irrevocable transactions and their applications, in: SPAA '08: Proceedings of the twentieth annual symposium on Parallelism in algorithms and architectures, ACM, New York, NY, USA, 2008, pp. 285-296. doi:http://doi.acm.org/10.1145/1378533.1378584.

[46] M. Spear, M. Michael, M. Scott, Inevitability mechanisms for software transactional memory, in: 3rd workshop of Transactional Computing (TRANSACT), 2008. 
[47] W. N. Scherer, III, M. L. Scott, Advanced contention management for dynamic software transactional memory, in: PODC '05: Proceedings of the twenty-fourth annual ACM symposium on Principles of distributed computing, ACM, New York, NY, USA, 2005, pp. 240-248. doi:http://doi.acm.org/10.1145/1073814.1073861.

[48] Y. Ni, A. Welc, A.-R. Adl-Tabatabai, M. Bach, S. Berkowits, J. Cownie, R. Geva, S. Kozhukow, R. Narayanaswamy, J. Olivier, S. Preis, B. Saha, A. Tal, X. Tian, Design and implementation of transactional constructs for $\mathrm{c} / \mathrm{c}++$, in: OOPSLA '08: Proceedings of the 23rd ACM SIGPLAN conference on Object-oriented programming systems languages and applications, ACM, New York, NY, USA, 2008, pp. 195-212. doi:http://doi.acm.org/10.1145/1449764.1449780.

[49] R. Guerraoui, M. Herlihy, B. Pochon, Toward a theory of transactional contention managers, in: PODC '05: Proceedings of the twenty-fourth annual ACM symposium on Principles of distributed computing, ACM, New York, NY, USA, 2005, pp. 258-264. doi:http://doi.acm.org/10.1145/1073814.1073863.

[50] M. J. Moravan, J. Bobba, K. E. Moore, L. Yen, M. D. Hill, B. Liblit, M. M. Swift, D. A. Wood, Supporting nested transactional memory in $\log$ TM, in: ASPLOSXII: Proceedings of the 12th international conference on Architectural support for programming languages and operating systems, ACM Press, New York, NY, USA, 2006, pp. 359-370. doi:http://doi.acm.org/10.1145/1168857.1168902.

[51] K. E. Moore, J. Bobba, M. J. Moravan, M. D. Hill, D. A. Wood, LogTM: Logbased transactional memory, in: Proceedings of the 12th International Symposium on High-Performance Computer Architecture, 2006, pp. 254-265.

[52] L. Hammond, V. Wong, M. Chen, B. D. Carlstrom, J. D. Davis, B. Hertzberg, M. K. Prabhu, H. Wijaya, C. Kozyrakis, K. Olukotun, Transactional memory coherence and consistency, in: Proceedings of the 31st Annual International Symposium on Computer Architecture, IEEE Computer Society, 2004, p. 102.

[53] B. D. Carlstrom, J. Chung, H. Chafi, A. McDonald, C. C. Minh, L. Hammond, 
C. Kozyrakis, K. Olukotun, Executing Java programs with transactional memory, Science of Computer Programming 63 (2006) 111-129.

[54] C. Blundell, J. Devietti, E. C. Lewis, M. M. K. Martin, Making the fast case common and the uncommon case simple in unbounded transactional memory, in: ISCA '07: Proceedings of the 34th Annual International Symposium on Computer architecture, ACM Press, New York, NY, USA, 2007, pp. 24-34. doi:http://doi.acm.org/10.1145/1250662.1250667.

[55] S. Kumar, M. Chu, C. J. Hughes, P. Kundu, A. Nguyen, Hybrid transactional memory, in: PPoPP '06: Proc. 11th symposium on Principles and Practice of Parallel Programming, ACM, New York, NY, USA, 2006, pp. 209-220. doi:http://doi.acm.org/10.1145/1122971.1123003.

[56] C. C. Minh, M. Trautmann, J. Chung, A. McDonald, N. Bronson, J. Casper, C. Kozyrakis, K. Olukotun, An effective hybrid transactional memory system with strong isolation guarantees, in: ISCA '07: Proceedings of the 34th Annual International Symposium on Computer architecture, ACM Press, New York, NY, USA, 2007, pp. 69-80. doi:http://doi.acm.org/10.1145/1250662.1250673.

[57] A. Shriraman, M. F. Spear, H. Hossain, V. J. Marathe, S. Dwarkadas, M. L. Scott, An integrated hardware-software approach to flexible transactional memory, in: ISCA '07: Proceedings of the 34th Annual International Symposium on Computer architecture, ACM Press, New York, NY, USA, 2007, pp. 104-115. doi:http://doi.acm.org/10.1145/1250662.1250676.

[58] R. Rajwar, M. Herlihy, K. Lai, Virtualizing transactional memory, in: ISCA '05: Proceedings of the 32nd Annual International Symposium on Computer Architecture, IEEE Computer Society, Washington, DC, USA, 2005, pp. 494-505. doi:http://dx.doi.org/10.1109/ISCA.2005.54.

[59] W. Chuang, S. Narayanasamy, G. Venkatesh, J. Sampson, M. V. Biesbrouck, G. Pokam, B. Calder, O. Colavin, Unbounded page-based transactional memory, in: ASPLOS-XII: Proc. 12th Int. conf. on Architectural Support for Programming 
Languages and Operating Systems, ACM Press, San Jose, California, USA, 2006, pp. 347-358. doi:http://doi.acm.org/10.1145/1168857.1168901.

[60] B. Saha, A.-R. Adl-Tabatabai, Q. Jacobson, Architectural support for software transactional memory, in: MICRO '06: Proceedings of the 39th Annual IEEE/ACM International Symposium on Microarchitecture, IEEE Computer Society, Washington, DC, USA, 2006, pp. 185-196. doi:http://dx.doi.org/10.1109/MICRO.2006.9.

URL http://csdl2. computer.org/persagen/DLAbsToc.jsp?resourc\% ePath=/dl/proceedings/\&toc=comp/proceedings/micro/2006/2732/ 00/2732toc.xml

[61] B. Goetz, Optimistic Thread Concurrency, http://www.azulsystems.com/ products/whitepaper/wp_otc.pdf (Jan. 2006).

[62] D. Dice, Y. Lev, M. Moir, D. Nussbaum, Early experience with a commercial hardware transactional memory implementation (2009) 157-168.

[63] H. Volos, A. J. Tack, N. Goyal, M. M. Swift, A. Welc, xCalls: safe I/O in memory transactions, in: EuroSys '09: Proc. 4th ACM European Conference on Computer Systems, 2009, pp. 247-260. 